Physics

Physics Research Publications

\title{
Effect of electron-beam irradiation on graphene field effect devices
}

\author{
I. Childres* \\ L. A. Jauregui ${ }^{\dagger}$
J. F. Tian**
R. Jalilian ${ }^{\dagger}$
I. Jovanovic ${ }^{\ddagger \ddagger}$
Y. P. $\mathrm{Chen}^{\S}$

M. Foxe

$\dagger$
$\ddagger$
$* *$
$\dagger \dagger$
$\ddagger \ddagger$
$\S$

This paper is posted at Purdue e-Pubs.

http://docs.lib.purdue.edu/physics_articles/1215 


\section{Effect of electron-beam irradiation on graphene field effect devices}

Isaac Childres, ${ }^{1,2}$ Luis A. Jauregui, ${ }^{2,3}$ Michael Foxe, ${ }^{4, a)}$ Jifa Tian, ${ }^{1,2}$ Romaneh Jalilian, ${ }^{1,2, b)}$ Igor Jovanovic, ${ }^{4, a)}$ and Yong P. Chen ${ }^{1,2,3, c)}$

${ }^{1}$ Department of Physics, Purdue University, West Lafayette, Indiana 47907, USA

${ }^{2}$ Birck Nanotechnology Center, Purdue University, West Lafayette, Indiana 47907, USA

${ }^{3}$ School of Electrical and Computer Engineering, Purdue University, West Lafayette, Indiana 47907, USA

${ }^{4}$ School of Nuclear Engineering, Purdue University, West Lafayette, Indiana 47907, USA

(Received 24 August 2010; accepted 27 September 2010; published online 27 October 2010)

Electron beam exposure is a commonly used tool for fabricating and imaging graphene-based devices. Here, we present a study of the effects of electron-beam irradiation on the electronic transport properties of graphene and the operation of graphene field-effect transistors (GFETs). Exposure to a $30 \mathrm{keV}$ electron-beam caused negative shifts in the charge-neutral point (CNP) of the GFET, interpreted as due to n-doping in the graphene from the interaction of the energetic electron beam with the substrate. The shift in the CNP is substantially reduced for suspended graphene devices. The electron beam is seen to also decrease the carrier mobilities and minimum conductivity, indicating defects created in the graphene. The findings are valuable for understanding the effects of radiation damage on graphene and for the development of radiation-hard graphene-based electronics. (c) 2010 American Institute of Physics. [doi:10.1063/1.3502610]

Graphene has been the focus of much research in material science and nanotechnology due to its unique properties and potentials in device applications. Many reports have been made on graphene's very high electrical conductivity ${ }^{1,2}$ at room temperature, and its potential use in next-generation transistors, ${ }^{3}$ nanosensors, ${ }^{4}$ and many other applications.

The effect of e-beam irradiation on graphene and graphene devices is important because of the prevalence of electron beams in both imaging of graphene, e.g., scanning electron microscopy (SEM) and transmission electron microscopy (TEM), and fabrication of graphene devices using e-beam lithography (EBL). In addition, such studies are important to develop radiation-hard graphene-based electronics that can stand up to extreme conditions such as charged particle irradiation in space. ${ }^{5}$

Several recent works in the field of energetic particle irradiation of graphene have used positive ions $^{6-11}$ or protons. ${ }^{12}$ It has been suggested that such irradiations create lattice defects in graphene. There have been studies using energetic electron-beam irradiation to create disorder in carbon nanotubes and graphite ${ }^{13}$ and others that focus mostly on the Raman spectroscopy of the electron-beam-induced defects in graphene. ${ }^{14-16}$ In this study, we present data on the effect of energetic electron-beam irradiation on the electrical transport properties of single-layer graphene and the operation of graphene field-effect transistors.

Our graphene samples are fabricated by micromechanical exfoliation ${ }^{1}$ of highly ordered pyrolytic graphite ("ZYA" grade, momentive performance materials) onto a $\mathrm{p}^{++}$(borondoped, with room temperature resistivity $<0.005 \Omega \mathrm{cm}) \mathrm{Si}$ wafer covered with $300 \mathrm{~nm}$ of $\mathrm{SiO}_{2}$. Single-layer graphene flakes, typically around $100 \mu \mathrm{m}^{2}$ in size, are identified using color contrast with an optical microscope, ${ }^{17}$ and then confirmed with Raman spectroscopy (using a $532 \mathrm{~nm}$ excitation

\footnotetext{
${ }^{a)}$ Present address: Department of Mechanical and Nuclear Engineering, The Pennsylvania State University, University Park, Pennsylvania 16802, USA.

b) Present address: NaugaNeedles, Louisville, Kentucky 40299, USA.

${ }^{c)}$ Electronic mail: yongchen@ purdue.edu.
}

laser). ${ }^{18}$ Graphene field-effect devices are subsequently fabricated using EBL. The electrical contacts (5-nm-thick chromium and 65-nm-thick gold) are fabricated by electron-beam evaporation.

A graphene device is placed in a scanning electron microscope (EVO40) under high vacuum $\left(10^{-6}\right.$ Torr). An area of $25 \times 25 \mu^{2}$ [shown in Fig. 1(a) inset as the blackbordered box indicated by the arrow], including the graphene flake on the device, is exposed to the electron beam. The electron beam's kinetic energy is $30 \mathrm{keV}$, the same energy that is used for our lithography and imaging processes. The beam current $\left(\mathrm{I}_{\mathrm{e}}\right)$ used ranges from 0.15 to $0.33 \mathrm{nA}$. The product of $I_{e}$ with accumulated exposure time $\left(T_{e}\right)$ gives the accumulated irradiation dosage (DOS) (e.g., $\mathrm{T}_{\mathrm{e}}=75 \mathrm{~s}$ and $\mathrm{I}_{\mathrm{e}}=0.15 \mathrm{nA}$ gives $\mathrm{DOS}=112.5 \mathrm{e}^{\left.-/ \mathrm{nm}^{2}\right)}$. In comparison, the typical exposure used in our lithography process is around $1 \mathrm{e}^{-} / \mathrm{nm}^{2}$. SEM imaging typically exposes samples to at least $100 \mathrm{e}^{-} / \mathrm{nm}^{2}$.
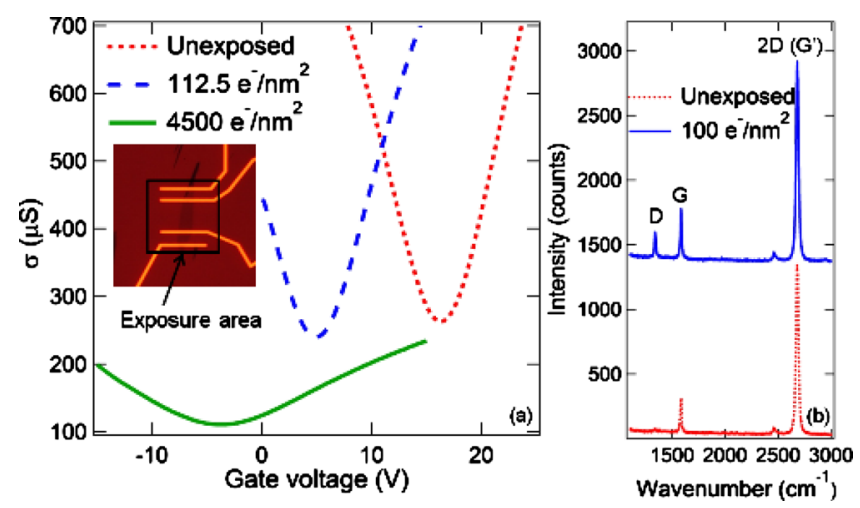

FIG. 1. (Color online) (a) Measured graphene conductivity as a function of a forward-sweeping back gate voltage after various doses of electron-beam irradiation for a graphene device on a $\mathrm{SiO}_{2} / \mathrm{Si}$ substrate (sample "A"). The source-drain current $\left(\mathrm{I}_{\mathrm{ds}}\right)$ used is $100 \mathrm{nA}$. The inset shows an optical image of the graphene sample measured. (b) Raman spectra before and after irradiation (dosage $=100 \mathrm{e}^{-} / \mathrm{nm}^{2}$, spectrum offset for clarity) on a similar graphene device (sample "B"). The wavelength of the excitation laser is 532 $\mathrm{nm}$. 
After each successive exposure, the graphene device is removed from the scanning electron microscope, and then room-temperature electrical or Raman measurements are promptly performed. Field-effect electrical measurements with the p-doped Si substrate as the back gate are performed using a probe station filled with argon gas at $1 \mathrm{~atm}$. Raman spectra are taken with a $532 \mathrm{~nm}$ excitation laser in an ambient atmosphere.

Results from three forward gate voltage sweeps (fieldeffect) measured from a representative device ("A") are shown in Fig. 1(a). The conductivity $(\sigma)$ is determined by four-terminal resistance measurements using low-frequency lock-in detection. Initially (before exposure), the device shows a charge-neutral "Dirac" point [charge-neutral point (CNP), defined as where $\sigma$ is at a minimum $\left.{ }^{1}\right]$ of $16.3 \mathrm{~V}$. The positive CNP is typical in our fabricated devices because of extrinsic hole doping in graphene from, e.g., water molecules in the air ${ }^{19}$ and resist residues from the lithography process. ${ }^{20}$ After the device is exposed to the electron beam with DOS $=112.5 \mathrm{e}^{-} / \mathrm{nm}^{2}$, we observe an appreciable negative shift in the CNP to $4.9 \mathrm{~V}$.

After a larger DOS $=4500 \mathrm{e}^{-} / \mathrm{nm}^{2}$ (accumulated from multiple exposures), the CNP decreases further to $-3.8 \mathrm{~V}$, and the slope of the field-effect curve (away from the CNP), related directly to the carrier mobility, decreases significantly in magnitude. The minimum conductivity $\left(\sigma_{\text {min }}\right.$, taken as $\sigma$ at the CNP) also decreases substantially.

Figure 1(b) shows Raman spectra on a similar sample. We observe the appearance of the disorder-induced " $D$ " peak after electron-beam irradiation. This is similar to what was observed previously, ${ }^{14-16}$ indicating defects created by electron-beam irradiation in graphene.

In this work, we focus on the effect of electron-beam irradiation on electronic transport properties. Figure 2 shows the CNP, mobilities and $\sigma_{\min }$ of sample "A" for a series of increasing irradiation dosages. Each of these quantities is calculated as the average of two measurements from forward and backward gate voltage sweeps.

The electron and hole mobilities $\left(\mu_{\mathrm{e}}\right.$ and $\mu_{\mathrm{h}}$, respectively) are extracted by examining the slope of the fieldeffect curve, conductivity $(\sigma)$ versus back gate voltage $\left(\mathrm{V}_{\mathrm{g}}\right)$, where $\mathrm{V}_{\mathrm{g}}$ is sufficiently far away from the CNP and the curve is in the linear regime using,

$$
\mu=(\mathrm{t} / \varepsilon) \times\left(\mathrm{d} \sigma / \mathrm{dV}_{\mathrm{g}}\right),
$$

where $\mathrm{t}=300 \mathrm{~nm}$ is the thickness of the $\mathrm{SiO}_{2}$ and $\varepsilon=3.9$ $\times \varepsilon_{0}=3.45 \times 10^{-11} \mathrm{~F} / \mathrm{m}$ is the permittivity of the $\mathrm{SiO}_{2}{ }^{19}$ During the first several irradiations, the mobilities decrease sharply from $\sim 5000-6000 \mathrm{~cm}^{2} / \mathrm{V} \mathrm{s}$ (pre-exposure), then begin to saturate at $\sim 1000 \mathrm{~cm}^{2} / \mathrm{V} \mathrm{s}$ after $\sim 1000 \mathrm{e}^{-} / \mathrm{nm}^{2}$ of accumulated exposure. We also see $\sigma_{\min }$, plotted in units of $e^{2} / h$ (where $e$ is electron charge and $h$ is Planck's constant), decreases by more than a factor of two, from $\sim 7 e^{2} / h$ before exposure to $\sim 3 e^{2} / h$.

The CNP decreases from $\sim 17 \mathrm{~V}$ before exposure to less than $0 \mathrm{~V}$ after extended exposure. We interpret most of the negative shift in the CNP as due to the interaction of the $\mathrm{SiO}_{2} / \mathrm{Si}$ substrate with energetic electron-beam irradiation. This irradiation generates electron-hole pairs, and the lessmobile holes can get trapped at the $\mathrm{SiO}_{2} / \mathrm{Si}$ interface to create an effective extra positive bias, attracting electrons in the graphene and resulting in a decreased CNP. This is similar to

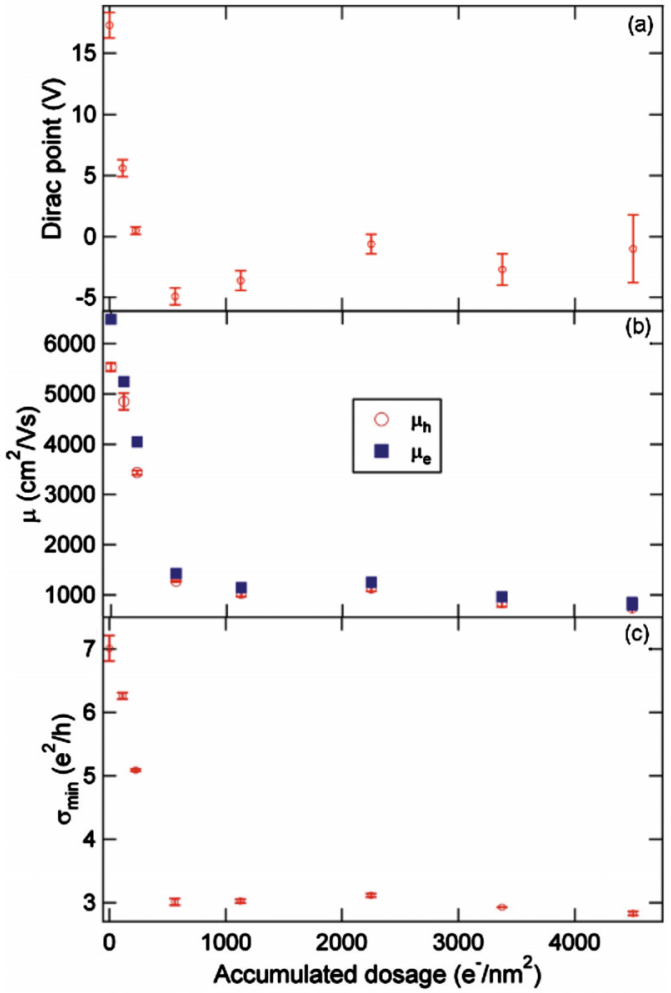

FIG. 2. (Color online) Charge-neutral "Dirac" point (a), electron and hole field-effect mobilities (b), and minimum conductivity (c) of sample "A" as functions of accumulated electron-beam irradiation dosage. Each data point is the average of two measurements from forward and backward gate voltage sweeps. The error bars reflect the variation between the two sweeps.

the negative shift in threshold voltage well-known in irradiated metal-oxide-semiconductor field-effect transistors. ${ }^{5}$ Control studies performed using "simulated" exposure procedures (similar to those used in Fig. 2), but without actually turning on the electron-beam, show a much smaller negative shift (by $\sim 5 \mathrm{~V}$, compared to $\sim 20 \mathrm{~V}$ of shift with the electron beam on) of the CNP (possibly because the SEM vacuum pumping helps remove surface adsorbates on graphene) and negligible changes in $\mu_{\mathrm{e}}, \mu_{\mathrm{h}}$, and $\sigma_{\min }$.

To further investigate the influence of the substrate on graphene's CNP under energetic electron-beam irradiation, we have also fabricated suspended graphene devices and irradiated them in the same manner. ${ }^{21}$ All field-effect measurements on the suspended devices are two-terminal. Figure 3(a) shows the CNP decrease by less than $0.16 \mathrm{~V}$ after DOS $=112.5 \mathrm{e}^{-} / \mathrm{nm}^{2}$ (compared to a $\sim 12 \mathrm{~V}$ shift for our typical substrate-supported device). This confirms the importance of the substrate for the observed CNP shift. The negative CNP shift we observed was not seen in positive ion irradiation studies where the ion kinetic energy was much lower (e.g., $500 \mathrm{eV}$ ). ${ }^{9}$

The substantial drop in the mobilities and the characteristic appearance of the Raman " $\mathrm{D}$ " band [also observed in suspended graphene, Fig. 3(b)] in the graphene samples after exposure indicate that electron beam irradiation is damaging the graphene lattice structure, creating defects that also scatter the carriers.

In summary, we have observed primarily two effects of electron-beam irradiation on graphene field-effect devices. The CNP of the substrate-supported graphene decreased significantly, indicating a doping of the graphene caused by the 


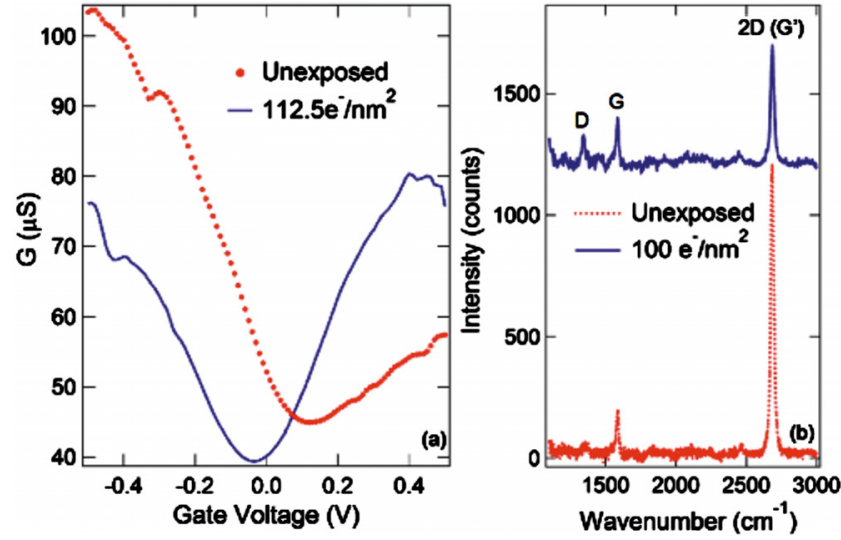

FIG. 3. (Color online) (a) Measured two-terminal conductance as a function of back gate voltage before and after electron-beam irradiation (dosage $=112.5 \mathrm{e}^{-} / \mathrm{nm}^{2}$ ) for a suspended graphene device (sample "C"). The $\mathrm{I}_{\mathrm{ds}}$ used is $100 \mathrm{nA}$. (b) Raman spectra (excitation wavelength $=532 \mathrm{~nm}$ ) taken before and after irradiation (dosage $=100 \mathrm{e}^{-} / \mathrm{nm}^{2}$, spectrum offset for clarity) on a similar suspended graphene device (sample "D").

interaction of the energetic electron beam and the substrate. Also, the graphene mobility decreased significantly and a "D" peak emerged in the Raman spectra, indicating irradiation-induced defects in graphene. Care should be taken when using SEM and TEM to image and EBL to fabricate graphene devices, as extended exposure could result in a degradation of the graphene device's electrical transport properties. On the other hand, the change caused in the CNP is highly dependent on the interaction with the sample substrate and suspended graphene devices would be excellent candidates for use in rad-hard electronics.

This work has been partially supported by National Science Foundation (Grant No. ECCS-0833689), Department of Homeland Security (Grant No. 2009-DN-077-15 ARI03602), and by the Defense Threat Reduction Agency (Grant No. HDTRA1-09-1-0047). We thank Leonid Rohkinson for the access to the SEM. Yong P. Chen also acknowledges support from the Miller Family Endowment and IBM.

${ }^{1}$ A. K. Geim and K. S. Novoselov, Nature Mater. 6, 183 (2007).

${ }^{2}$ C. Berger, Z. Song, X. Li, X. Wu, N. Brown, C. Naud, D. Mayou, T. Li, J. Hass, A. N. Marchenkov, E. H. Conrad, P. N. First, and W. A. de Heer, Science 312, 1191 (2006).

${ }^{3}$ F. Schwierz, Nat. Nanotechnol. 5, 487 (2010).

${ }^{4}$ F. Schedin, A. K. Geim, S. V. Morozov, E. W. Hill, P. Blake, M. I. Katsnelson, and K. S. Novoselov, Nature Mater. 6, 652 (2007)

${ }^{5}$ C. Claeys and E. Simoen, Radiation Effects in Advanced Semiconductor Materials and Devices (Springer, Berlin, 2002).

${ }^{6}$ G. Compagnini, F. Giannazzo, S. Sonde, V. Raineri, and E. Rimini, Carbon 47, 3201 (2009).

${ }^{7}$ M. M. Lucchese, F. Stavale, E. H. Ferreira, C. Vilani, M. V. O. Moutinho, R. B. Capaz, C. A. Achete, and A. Jorio, Carbon 48, 1592 (2010).

${ }^{8}$ L. Tapasztó, G. Dobrik, P. Nemes-Incze, G. Vertesy, P. Lambii, and L. P. Biro, Phys. Rev. B 78, 233407 (2008).

${ }^{9}$ J.-H. Chen, W. G. Cullen, C. Jang, M. S. Fuhrer, and E. D. Williams, Phys. Rev. Lett. 102, 236805 (2009).

${ }^{10}$ J. J. Lopez, F. Greer, and J. R. Greer, J. Appl. Phys. 107, 104326 (2010)

${ }^{11}$ C. L. Tan, Z. B. Tan, L. Ma, F. M. Qu, F. Yang, J. Chen, G. T. Liu, H. F. Yang, C. L. Yang, and L. Lu, Sci. China, Ser. G 52, 1293 (2009).

${ }^{12}$ A. Arndt, D. Spoddig, P. Esquinazi, J. Barzola-Quiquia, S. Durari, and T. Butz, Phys. Rev. B 80, 195402 (2009).

${ }^{13}$ A. V. Krasheninnikov and K. Nordlund, J. Appl. Phys. 107, 071301 (2010).

${ }^{14}$ D. Teweldebrhan and A. A. Balandin, Appl. Phys. Lett. 94, 013101 (2009).

${ }^{15}$ G. Rao, S. Mctaggart, J. L. Lee, and R. E. Geer, Electron Crystallography for Materials Research and Quantitative Characterization of Nanostructured Materials (Mater. Res. Soc. Symp. Proc. 1184, Warrendale, PA, 2009), p. HH03-07.

${ }^{16} \mathrm{M}$. Xu, D. Fujita, and N. Hanagata, Nanotechnology 21, 265705 (2010).

${ }^{17}$ P. Blake, E. W. Hill, A. H. Castro Neto, K. S. Novoselov, D. Jiang, R. Yang, T. J. Booth, and A. K. Geim, Appl. Phys. Lett. 91, 063124 (2007)

${ }^{18}$ A. C. Ferrari, J. C. Meyer, V. Scardaci, C. Casiraghi, M. Lazzeri, F. Mauri, S. Piscanec, D. Jiang, K. S. Novoselov, S. Roth, and A. K. Geim, Phys Rev. Lett. 97, 187401 (2006)

${ }^{19}$ K. S. Novoselov, A. K. Geim, S. V. Morozov, D. Jiang, Y. Zhang, S. V. Dubonos, I. V. Grigorieva, and A. A. Firsov, Science 306, 666 (2004).

${ }^{20}$ R. Jalilian, L. A. Jauregui, G. Lopez, J. Tian, C. Roecker, M. M. Yazdanpanah, R. W. Cohn, I. Jovanovic, and Y. P. Chen, arXiv:1003.5404v1 (unpublished).

${ }^{21}$ To suspend the graphene devices, we dip the device in a buffered oxide etchant for $1 \mathrm{~min}$, rinse the etchant with acetone and isopropyl alcohol (IPA), then dry the IPA with a critical-point dryer to reduce surface tensions. 